\title{
A firm, pea-shaped lesion on the paw of a beagle
}

A pharmaceutical company where I serve as the attending veterinarian uses male and female pure-bred beagles for pharmacodynamic/pharmacokinetic and long-term toxicology studies. The laboratory animal group at one point purchased juvenile beagles aged 8-12 months. The company houses the dogs socially in small groups of two to four beagles matched for gender and age. Environmental and housing conditions comply with the Guide for the Care and Use of Laboratory Animals and the Animal Welfare Act Regulations.

During a routine clinical examination of a 12-month-old male dog, a veterinary technician noted a 2-3-mm diameter lesion in the fourth interdigital space of the left front limb (Fig. 1). The dog weighed $12.2 \mathrm{~kg}$ and had no history of disease. It was a large beagle, both in height and bodyweight for its

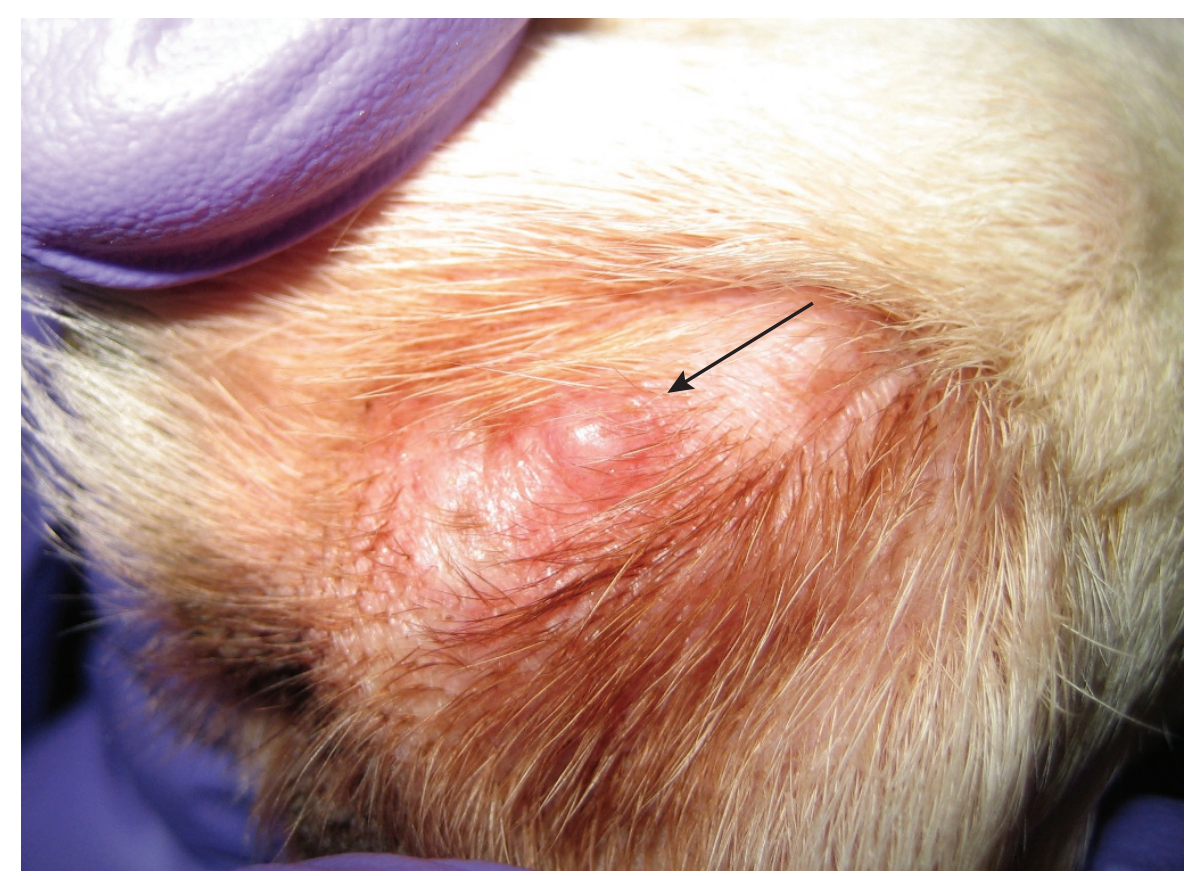

FIGURE 1 | A 2-3 mm-diameter lesion in the fourth interdigital space of the left front paw limb of a 12-month old male research beagle. age (probably in the top fifth percentile), but was lean and in good body condition. The lesion was in the skin web of the interdigital space. On palpation, the lesion felt firm and did not appear to be painful to the dog. The dog had no clinical signs and the lesion was considered an incidental finding.

Over the next few days, animal technicians noted the dog licking its front left paw. On further examination three days later, the lesion was slightly larger $(\sim 4-5 \mathrm{~mm})$ and the dog showed discomfort when the lesion was palpated. When I examined the dog, I found it was bright, alert, and responsive; its body temperature, heart rate, respiratory rate, mucous membrane color, and capillary refill time were within normal limits. The dog could walk. The lesion was 5-6 $\mathrm{mm}$ in diameter and the surrounding web skin was slightly erythematous. The

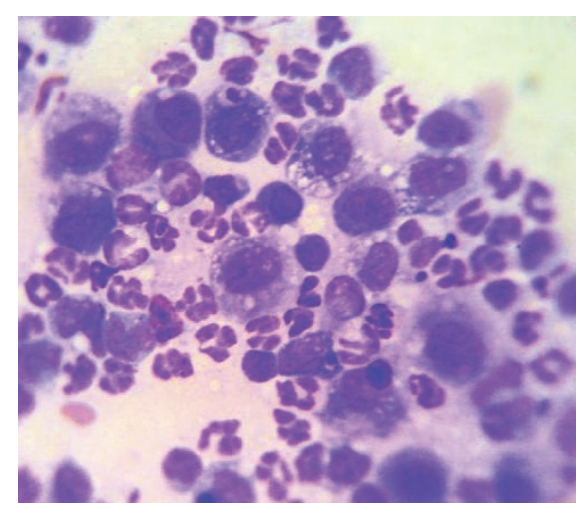

FIGURE 2 | Photomicrograph of a fine needle aspirate of the lesion in Figure 1. The aspirate was taken 5 days after the photograph in Figure 1, when the lesion was 5-6 mm in diameter. There is a mixed population of inflammatory cells composed of toxic and degenerating neutrophils, and phagocytically active macrophages. Rare lymphocytes, plasma cells, and red blood cells are also present. (Modified Wright-Giemsa stain, high power magnification).

dog showed discomfort when I palpated the lesion and mild-to-moderate pain when I gently squeezed the lesion.

I took a fine-needle aspirate of the lesion with a 25-gauge needle. The lesion bled, but mild pressure applied to the site of puncture stopped the bleeding. The aspirate contained serosanguineous fluid. Using an in-house modified Romanovsky stain (Diff-Quik, Dade Behring, Inc., Deerfield, IL) I stained an air-dried sample of the aspirate (Fig. 2). On microscopic examination, I saw some neutrophils and macrophages and many red blood cells. I did not see any microorganisms.

Based on the history, clinical findings, and gross appearance of the lesion, what do you think it is? Is this lesion common in research beagles? What is the cause of the lesion? How would you treat it?

What's your diagnosis? 